This is the author's version of a work that was accepted for publication in International Mathematics Research Notices. Changes resulting from the publishing process, such as peer review, editing, corrections, structural formatting, and other quality control mechanisms may not be reflected in this document. Changes may have been made to this work since it was submitted for publication. A definitive version was subsequently published in

Int. Math. Res. Notices $2007 \mathrm{rnm} 032$.

http://dx.doi.org/10.1093/imrn/rnm032 


\title{
An extension of James's Conjecture
}

\author{
Matthew Fayers* \\ Queen Mary, University of London, Mile End Road, London E1 4NS, U.K. ${ }^{\dagger}$ \\ Massachusetts Institute of Technology, 77 Massachusetts Avenue, Cambridge MA 02139-4307, U.S.A. ${ }^{\ddagger}$ \\ m.fayers@qmul.ac.uk
}

2000 Mathematics subject classification: 20C30, 20C08, 20C33, 20G05

\begin{abstract}
Let $B$ be a block of an Iwahori-Hecke algebra or $q$-Schur algebra of the symmetric group. The decomposition matrix for $B$ may be obtained from the decomposition matrix of the corresponding block $B^{\prime}$ in infinite characteristic by post-multiplying by an adjustment matrix; since (by a deep theorem of Ariki) there is an algorithm for computing the decomposition matrix for $B^{\prime}$, the hard part of the decomposition number problem for $B$ is to find the adjustment matrix. James's Conjecture suggests a sufficient condition for this adjustment matrix to be the identity matrix. We extend James's Conjecture to give a necessary and sufficient condition, and prove the necessity of our condition.
\end{abstract}

\section{Introduction}

Let $\mathbb{F}$ be any field, and $q$ an invertible element of $\mathbb{F}$. Given a non-negative integer $n$, let $\mathcal{H}_{n}=$ $\mathcal{H}_{\mathbb{F}, q}\left(\Im_{n}\right)$ denote the Iwahori-Hecke algebra of the symmetric group $\Xi_{n}$; this is a 'deformation' of the group algebra $\mathrm{F}_{n}$ which arises in various mathematical contexts.

If $q$ is not a root of unity in $\mathbb{F}$, then $\mathcal{H}_{n}$ is semi-simple; if $q$ is a root of unity, then the representation theory is very similar to the $p$-modular representation theory of $\mathfrak{S}_{n}$, with the rôle of the prime $p$ being played by the smallest integer $e$ for which $1+q+\cdots+q^{e-1}=0$ in $\mathbb{F}$. The most important problem in the representation theory of $\mathcal{H}_{n}$ is to determine the decomposition numbers, i.e. the composition multiplicities of the irreducible modules in the Specht modules; in the case $q=1$ (where $\mathcal{H}_{n}$ is simply the group algebra $\mathbb{F}_{n}$ ), these are the decomposition numbers in the usual representation-theoretic sense. This problem remains open in general, but has been solved in some special cases. In particular, if $\mathbb{F}$ has infinite characteristic (we use the convention that the characteristic of $\mathbb{F}$ is the order of the prime subfield of $\mathbb{F}$ ) then the decomposition numbers can be computed. This result is due to Ariki [1], verifying a conjecture of Lascoux, Leclerc and Thibon; an explicit algorithm (the 'LLT algorithm') for computing the decomposition matrices is given in [20], and a discussion of Ariki's proof appears in the article by Geck [15].

${ }^{*}$ This research was undertaken with the support of a Research Fellowship from the Royal Commission for the Exhibition of 1851 . The author is very grateful to the Commission for its generous support.

${ }^{+}$Correspondence address.

¥This research was undertaken while the author was visiting M.I.T. as a Postdoctoral Fellow. He is very grateful to Prof. Richard Stanley for the invitation, and to M.I.T. for its hospitality. 
If $\mathbb{F}$ has finite characteristic, then the infinite characteristic result can still be used: if we take a primitive $e$ th root of unity $\zeta$ in $\mathbb{C}$, then the decomposition matrix for $\mathcal{H}_{n}$ may be obtained from the decomposition matrix for $\mathcal{H}_{\mathbb{C}, \zeta}\left(\mathbb{S}_{n}\right)$ by post-multiplying by a certain square matrix with nonnegative integer entries called the adjustment matrix for $\mathcal{H}_{n}$. So in effect the problem of calculating the decomposition matrix of $\mathcal{H}_{n}$ is equivalent to calculating its adjustment matrix.

The same situation applies to the representation theory of the $q$-Schur algebra. This is a deformation of the Schur algebra, a certain finite-dimensional algebra which encodes the theory of polynomial representations of the general linear group. Again, there is an algorithm for computing the decomposition numbers of the $q$-Schur algebra if the underlying field has infinite characteristic; this algorithm was given by Leclerc and Thibon [22], and the proof that it works is due to Varagnolo and Vasserot [26]. And as with the Iwahori-Hecke algebras, one may obtain the decomposition matrix for a $q$ Schur algebra in finite characteristic by using an adjustment matrix. In fact, the adjustment matrix for the Iwahori-Hecke algebra occurs as a submatrix of that for the $q$-Schur algebra, so computing the adjustment matrices for $q$-Schur algebras is sufficient to compute those for the Iwahori-Hecke algebras.

One can simplify the calculation of adjustment matrices by considering individual blocks of these algebras. However, there are very few blocks for which the adjustment matrices have been calculated. The most general statement we have about adjustment matrices is James's Conjecture. This remains unproved, but suggests that in certain cases (specifically, if the weight $w$ of a block is strictly less than the underlying characteristic $p$ ) then the adjustment matrix for that block should be the identity matrix. For Iwahori-Hecke algebras, this conjecture has been verified for blocks of weight at most four, thanks to the work of Richards [24] and the author [10,11].

In this paper, we extend James's Conjecture by asking whether the condition $w<p$ is necessary for a block to have adjustment matrix equal to the identity matrix. We shall see that for the $q$-Schur algebras this is the case, while for Iwahori-Hecke algebras it is not. We conjecture a necessary and sufficient condition for a block to have non-trivial adjustment matrix, and prove that the condition is necessary.

In the next section, we outline the background material we shall need. In Section 3, we give our conjecture. In Sections 4 and 5 we prove our conjecture in one direction for the Iwahori-Hecke algebra, and in Section 6 we do the same for the $q$-Schur algebra. Finally in Section 7 we discuss a conjectured (but refuted) algorithm of Rouquier for computing decomposition matrices, and show that our results give more counterexamples.

\section{Background}

\subsection{Iwahori-Hecke algebras and $q$-Schur algebras}

An essential reference for the representation theory of the Iwahori-Hecke algebra and the $q$-Schur algebra is Mathas's book [23]; we summarise the results we need from this, together with some more recent work.

From now on we fix a field $\mathbb{F}$, and a root of unity $q$ in $\mathbb{F}$. We let $e$ be the smallest positive integer such that $1+q+\cdots+q^{e-1}=0$ in $\mathbb{F}$; thus, $e$ is the characteristic of $\mathbb{F}$ if $q=1$, and otherwise $e$ is the multiplicative order of $q$ in $\mathbb{F}$. We let $\mathcal{H}_{n}$ denote the Iwahori-Hecke algebra $\mathcal{H}_{\mathbb{F}, q}\left(\Im_{n}\right)$ of the symmetric group over $\mathbb{F}$ with parameter $q$, and $\mathcal{S}_{n}$ the $q$-Schur algebra $\mathcal{S}_{\mathbb{F}, q}(n, n)$; these are defined in [23]. When $q=1$, these algebras are simply the group algebra $\mathbb{F}_{n}$ and the classical Schur algebra over $\mathbb{F}$.

As usual, a partition of $n$ is a non-increasing sequence $\lambda=\left(\lambda_{1}, \lambda_{2}, \ldots\right)$ of non-negative integers whose sum is $n$; we write the unique partition of 0 as $\varnothing$. For each partition $\lambda$ of $n$, one defines a Specht 
module $S^{\lambda}$ for $\mathcal{H}_{n}$ and a Weyl module $W^{\lambda}$ for $\mathcal{S}_{n}$. Note that in this paper, we use the Specht and Weyl modules defined by Dipper and James [6, 7], rather than those in [23]; following [19], we re-label the Weyl modules using conjugate partitions, so that $W^{\lambda}$ is the module usually denoted $W^{\lambda^{\prime}}$ or $\Delta\left(\lambda^{\prime}\right)$. If $\lambda$ is $e$-regular (that is, if it does not have $e$ equal positive parts), then $S^{\lambda}$ has an irreducible cosocle $D^{\lambda}$; the modules $D^{\lambda}$ are non-isomorphic and give a complete set of irreducible $\mathcal{H}_{n}$-modules as $\lambda$ ranges over the set of $e$-regular partitions of $n$. Every Weyl module $W^{\lambda}$ has an irreducible cosocle $L^{\lambda}$; the modules $L^{\lambda}$ are non-isomorphic and give a complete set of irreducible $\mathcal{S}_{n}$-modules as $\lambda$ ranges over the set of partitions of $n$.

The central problem in this area is to compute the decomposition numbers for $\mathcal{H}_{n}$ and $\mathcal{S}_{n}$, i.e. the composition multiplicities $\left[S^{\lambda}: D^{\mu}\right]$ and $\left[W^{\lambda}: L^{\mu}\right]$. The following result, in which $\triangleq$ denotes the usual dominance order on partitions, comes from the fact that $\mathcal{H}_{n}$ and $\mathcal{S}_{n}$ are cellular algebras, as defined by Graham and Lehrer [16].

Theorem 2.1. Suppose $\lambda$ and $\mu$ are partitions of $n$.

1. If $\mu$ is e-regular, then $\left[S^{\mu}: D^{\mu}\right]=1$, while $\left[S^{\lambda}: D^{\mu}\right]=0$ unless $\mu \unrhd \lambda$.

2. $\left[W^{\mu}: L^{\mu}\right]=1$, while $\left[W^{\lambda}: L^{\mu}\right]=0$ unless $\mu \geqslant \lambda$.

In fact, the two decomposition number problems are intimately related via the following theorem, which is proved using the Schur functor [23, pp. 63-5].

Theorem 2.2. Suppose $\lambda$ and $\mu$ are partitions of $n$ with $\mu$ e-regular. Then

$$
\left[S^{\lambda}: D^{\mu}\right]=\left[W^{\lambda}: L^{\mu}\right]
$$

\subsection{Blocks and the abacus}

The decomposition number problem is made easier by considering blocks of $\mathcal{H}_{n}$ and $\mathcal{S}_{n}$ individually. Each Specht module lies in a single block of $\mathcal{H}_{n}$, and we abuse notation by saying that a partition $\lambda$ lies in a block to mean that $S^{\lambda}$ lies in that block. Conversely, each block contains at least one Specht module, so in order to describe the blocks of $\mathcal{H}_{n}$, it suffices to give a combinatorial criterion saying when two partitions lie in the same block. The same statement is true for Weyl modules and blocks of $\mathcal{S}_{n}$, and in fact the criterion on partitions is the same.

This combinatorial criterion is most conveniently described using the abacus. Given a partition $\lambda$ of $n$, let $r$ be a large integer and define the beta-numbers

$$
\beta_{i}=\lambda_{i}+r-i
$$

for $i=1, \ldots, r$. Now take an abacus with $e$ vertical runners, and mark positions $0,1, \ldots$ reading from left to right along successive rows. Place a bead at position $\beta_{i}$ for each $i$. The resulting configuration is called an abacus display for $\lambda$.

By sliding all the beads up their runners as far as they will go, we obtain the abacus display of a new partition, which is called the (e-)core of $\lambda$. This is a partition of $n-e w$, where $w$ is the (e-)weight of $\lambda$, i.e. the total distance moved by all the beads.

Theorem 2.3. Suppose $\lambda$ and $\mu$ are partitions of $n$. Then $\lambda$ and $\mu$ lie in the same block of $\mathcal{H}_{n}$ (or equivalently of $\mathcal{S}_{n}$ ) if and only if they have the same e-core.

Clearly any two partitions of $n$ with the same core will have the the same weight, and so we may speak of the weight and core of a block of $\mathcal{H}_{n}$ or $\mathcal{S}_{n}$. The weight of a block plays a vital rôle in James's Conjecture, as we shall see below. 
Later, we shall need to consider whether two partitions lying in a block $B$ have the same $\mathrm{em}$-core as well as the same $e$-core, for a particular integer $m$. From the above discussion, $\lambda$ and $\mu$ have the same $\mathrm{em}$-core if and only if we can get from the set of beta-numbers for $\lambda$ to the set of beta-numbers for $\mu$ by increasing and decreasing individual beta-numbers by integer multiples of $\mathrm{em}$. On an abacus with $e$ runners, this corresponds to moving beads up and down their runners through (multiples of) $m$ positions. For example, from the abacus displays for the partitions $\lambda, \mu, v$ below, we see that all three have the same 2-core, and that $\lambda$ and $\mu$ have the same 4-core, while $v$ has a different 4-core:

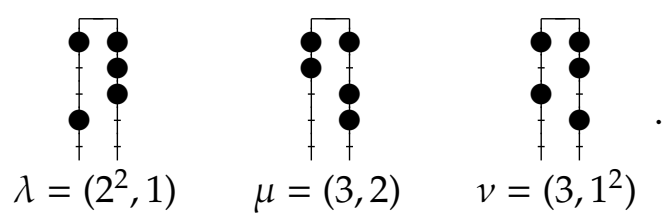

\subsection{Adjustment matrices and James's Conjecture}

In this section, we consider the case where the characteristic of $\mathbb{F}$ is finite. Given positive integers $n$ and $f$, let $\mathcal{H}_{n}^{f}$ be the Iwahori-Hecke algebra $\mathcal{H}_{\mathbb{G}, \zeta}\left(\Xi_{n}\right)$ with $\mathbb{G}$ a field of infinite characteristic and $\zeta$ a primitive $f$ th root of unity in G. Given partitions $\lambda$ and $\mu$ of $n$ with $\mu f$-regular, define $d_{\lambda \mu}^{f}$ to be the decomposition number $\left[S^{\lambda}: D^{\mu}\right]$ for $\mathcal{H}_{n}^{f}$. This number may be computed using the LLT algorithm, and in particular does not depend on the choice of $\mathbb{G}$ or $\zeta$. Let $D^{(f)}$ denote the decomposition matrix of $\mathcal{H}_{n}^{f}$, with rows indexed by partitions of $n$ and columns indexed by $f$-regular partitions of $n$, and with the $(\lambda, \mu)$-entry being $d_{\lambda \mu}^{f}$.

Theorem 2.4. Let $D$ be the decomposition matrix of $\mathcal{H}_{n}$, with rows indexed by partitions of $n$ and columns indexed by e-regular partitions of $n$. Then there is a square matrix $A$ with rows and columns indexed by e-regular partitions of $n$ and with non-negative integer entries, such that $D=D^{(e)} A$.

This result was first proved by Geck [14], and arises from a consideration of decomposition maps; an excellent introduction to this area can be found in Geck's article [15]. The matrix $A$ is simply the decomposition matrix corresponding to a decomposition map from $R_{0}\left(\mathcal{H}_{n}^{e}\right)$ to $R_{0}\left(\mathcal{H}_{n}\right)$ (where we use $R_{0}(H)$ to denote the Grothendieck group of any algebra $H$ ), and is referred to as the adjustment matrix of $\mathcal{H}_{n}$. Theorem 2.4 arises from the fact [15, Proposition 2.6] that the decomposition map from the Grothendieck group of a semi-simple Iwahori-Hecke algebra to $R_{0}\left(\mathcal{H}_{n}\right)$ factors via $R_{0}\left(\mathcal{H}_{n}^{e}\right)$, given suitable choices of $\mathbb{F}$ and $\mathbb{G}$.

It follows from Theorem 2.1 that the $(\mu, \mu)$-entry of $A$ is 1 for any $\mu$, while the $(\mu, v)$-entry is 0 unless $v \unrhd \mu$. Thus, if the order of the rows and columns is chosen appropriately, $A$ is a lower unitriangular matrix. Theorem 2.3 implies that $\mathcal{H}_{n}$ and $\mathcal{H}_{n}^{e}$ have the same block structure, and this implies that that the $(\mu, v)$-entry of $A$ is zero unless $\mu$ and $v$ lie in the same block of $\mathcal{H}_{n}$. So by restricting attention only to $e$-regular partitions lying in a given block $B$, one may define the adjustment matrix $A_{B}$ for $B$. One then gets $D_{B}=D_{B}^{(e)} A_{B}$, where $D_{B}$ is the decomposition matrix for $B$ and $D_{B}^{(e)}$ is the decomposition matrix for the block of $\mathcal{H}_{n}^{e}$ having the same core as $B$.

The situation for $q$-Schur algebras is much the same. With $\mathbb{G}$ and $\zeta$ as above, we define $\mathcal{S}_{n}^{f}$ to be the $\zeta$-Schur algebra over $\mathbb{G}$. For partitions $\lambda$ and $\mu$ of $n$, we let $d_{\lambda \mu}^{f}$ equal the decomposition number $\left[W^{\lambda}: L^{\mu}\right]$ for $\mathcal{S}_{n}^{f}$; note that according to our convention for labelling Weyl modules, the definition of $d_{\lambda \mu}^{f}$ agrees with that above when $\mu$ is $e$-regular. Let $\mathrm{D}^{(f)}$ denote the matrix with rows and columns indexed by partitions of $n$, with entries $d_{\lambda \mu^{\prime}}^{f}$, and let D be the decomposition matrix of $\mathcal{S}_{n}$. Then there 
is a square matrix A with non-negative integer entries such that $\mathrm{D}=\mathrm{D}^{(f)} \mathrm{A}$. Again, one may define the adjustment matrix for a particular block of $\mathcal{S}_{n}$.

Now we can state James's Conjecture.

Conjecture 2.5. Suppose B is a block of $\mathcal{H}_{n}$ or of $\mathcal{S}_{n}$ of weight $w<\operatorname{char}(\mathbb{F})$. Then the adjustment matrix for $B$ is the identity matrix.

Theorem 2.2 implies that the adjustment matrix for a block of $\mathcal{H}_{n}$ occurs as a submatrix of the adjustment matrix for the corresponding block of $\mathcal{S}_{n}$ (i.e. the block with the same core), so James's Conjecture for $q$-Schur algebras implies the same for Iwahori-Hecke algebras.

The question we wish to address in this paper is whether the condition $w<\operatorname{char}(\mathbb{F})$ is necessary for the adjustment matrix of a block of weight $w$ to be the identity. For the $q$-Schur algebras, we shall see that this is indeed the case. But for the Iwahori-Hecke algebras, it is not, as the first possible example - the unique 2-block of the symmetric group $\mathfrak{S}_{4}$ - shows. We shall give a conjectured necessary and sufficient criterion for the adjustment matrix of a block to be the identity matrix, and prove the necessity of our criterion. Of course, proving the sufficiency entails proving James's Conjecture, and we do not address this here.

In order to prove our results, we need to introduce additional 'adjustment matrices'. Using the set-up in [15, §3], one can obtain the following generalisation of Theorem 2.4.

Theorem 2.6. Suppose $p=\operatorname{char}(\mathbb{F})$ is finite, let $i$ be any non-negative integer, and let $f=e p^{i}$. Let $D$ be the decomposition matrix of $\mathcal{H}_{n}$. Then there is a matrix $A$ with non-negative integer entries, with rows indexed by $f$-regular partitions of $n$ and columns indexed by e-regular partitions of $n$, such that $D=D^{(f)} A$.

We refer to the matrix $A$ in this theorem as the $\left(e p^{i}\right)$-adjustment matrix of $\mathcal{H}_{n}$. Since two partitions having the same $e p^{i}$-core necessarily have the same $e$-core, we find that the $(\mu, v)$-entry of $A$ must be zero unless $\mu$ and $v$ have the same $e$-core. Accordingly, we can define the $\left(e p^{i}\right)$-adjustment matrix for a block $B$, taking the columns indexed by the $e$-regular partitions in $B$, and the rows indexed by the $e p^{i}$-regular partitions in $B$.

Using Theorem 2.6, we shall be able to prove that certain blocks have non-trivial adjustment matrices by calculating only decomposition numbers in infinite characteristic, i.e. the numbers $d_{\lambda \mu}^{e p^{i}}$ for various values of $i$. The following will be a useful result in proving that an adjustment matrix is not the identity matrix.

Lemma 2.7. Suppose B is a block of $\mathcal{H}_{n}$ or $\mathcal{S}_{n}$.

1. Suppose $\lambda$ and $\mu$ are partitions in $B$, with $\mu$ being e-regular if $B$ is a block of $\mathcal{H}_{n}$. If $d_{\lambda \mu}^{e p^{i}}>d_{\lambda \mu}^{e}$ for some $i$, then the $(\xi, \mu)$-entry of the adjustment matrix for $B$ is non-zero, for some $\mu \triangleright \xi \unrhd \lambda$.

2. Suppose that $\lambda, \mu, v$ are distinct partitions in $B$, and that if $B$ is a block of $\mathcal{H}_{n}$ then $\mu$ is e-regular. Suppose also that $\lambda$ and $v$ have the same ep-core, and that there is no partition $\xi$ with this ep-core such that $\mu \unrhd \xi \triangleright v$. Suppose that $d_{v \mu}^{e}>0$, while $d_{\lambda v}^{e p}>d_{\lambda \mu}^{e}$. Then the adjustment matrix for B is not the identity matrix.

Proof. For this proof, write $d_{\lambda \mu}$ to mean either $\left[S^{\lambda}: D^{\mu}\right]$ or $\left[W^{\lambda}: L^{\mu}\right]$ as appropriate, and similarly for $d_{v \mu}$.

1. Theorem 2.6 implies that $d_{\lambda \mu} \geqslant d_{\lambda \mu^{\prime}}^{e p^{i}}$ so that $d_{\lambda \mu}>d_{\lambda \mu}^{e}$. So there must be some partition $\xi \neq \mu$ such that $d_{\lambda \xi}^{e}>0$ and the $(\xi, \mu)$-entry of the adjustment matrix is non-zero. These conditions imply $\mu \triangleright \xi \unrhd \lambda$, by Theorem 2.1. 
2. The conditions given imply that the ep-core of $\mu$ is different from the ep-core of $v$, and hence $d_{v \mu}^{e p}=0$. On the other hand, $d_{v \mu} \geqslant d_{v \mu}^{e}>0$, so there must be some $\xi$ such that $d_{v \xi}^{e p}>0$ and the $(\xi, \mu)$-entry of the $(e p)$-adjustment matrix for $B$ is non-zero. These conditions imply that $\mu \unrhd \xi \unrhd v$ with $\xi$ having the same $e p$-core as $v$, and the hypotheses of the lemma then give $\xi=v$. So the $(v, \mu)$-entry of the ep-adjustment matrix for $B$ is positive, which implies that $d_{\lambda \mu} \geqslant d_{\lambda v}^{e p}$. This gives $d_{\lambda \mu}>d_{\lambda \mu^{\prime}}^{e}$ so that the adjustment matrix is non-trivial.

\section{$2.4 \quad e$-quotients, Scopes pairs, pyramids and Rouquier blocks}

In this section we summarise some of the combinatorial notions commonly used in the representation theory of Iwahori-Hecke algebras and $q$-Schur algebras, which we need in order to state our extension of James's Conjecture. We give brief definitions in order to save space; examples can be seen in subsequent sections.

Theorem 2.3 tells us that if we have abacus displays for two partitions $\lambda$ and $\mu$ of $n$ using the same number of beads, then $\lambda$ and $\mu$ lie in the same block (of $\mathcal{H}_{n}$ or $\mathcal{S}_{n}$ ) if and only if they have the same numbers of beads on corresponding runners. So we may define the abacus for a block $B$ by simply specifying the number of beads on each runner without specifying their positions, and we may specify a block by its abacus and its weight. We also use a new notation for partitions, based on the abacus. First we number the runners of the abacus according to the number of beads on each runner: we impose a total order $<$ on the the runners by saying that $s<t$ for two runners $s$ and $t$ if either $t$ lies to the left of $s$ and has strictly more beads than $s$, or $t$ lies to the right of $s$ and has at least as many beads as $s$; now we number the runners $0,1, \ldots, e-1$ so that $0<1<\cdots<e-1$. Given a partition $\lambda$ in $B$, we define the $e$-quotient of $\lambda$ to be the $e$-tuple of partitions $(\lambda[0], \ldots, \lambda[e-1])$, where $\lambda[i]$ is the partition obtained by viewing runner $i$ on its own as if it were an abacus with only one runner. In other words, $\lambda[i]_{j}$ equals the number of empty spaces on runner $i$ above the $j$ th lowest bead on that runner. If the numbers of beads on the runners reading from left to right are $b_{0}, \ldots, b_{e-1}$, then we may write $\lambda$ as

$$
\left\langle 0_{\lambda[0]}, \ldots, e-1_{\lambda[e-1]} \mid b_{0}, \ldots, b_{e-1}\right\rangle ;
$$

we omit $i_{\lambda[i]}$ if $\lambda[i]=\varnothing$, and we may omit $b_{0}, \ldots, b_{e-1}$ if these are understood.

Now we discuss Scopes pairs briefly. Suppose that we have an abacus display for a block $B$ of $\mathcal{H}_{n}$ of weight $w$, and that some runner $k$ has exactly $\kappa$ more beads than the runner immediately to the right, where $\kappa>0$. Then there is a block $C$ of $\mathcal{H}_{n+\kappa}$ of weight $w$ with an abacus obtained from the given abacus for $B$ by interchanging runner $k$ and the runner immediately to the right. We say that $B$ and $C$ form a $[w: \kappa]$-pair. Blocks forming such 'Scopes pairs' have very similar representation theories; in fact, if $w \leqslant \kappa$ then two such blocks are Morita equivalent. What is important for us is the relationship between the adjustment matrices of two blocks forming a $[w: \kappa]$-pair. $\mathcal{H}_{n+\kappa}$ is a free $\mathcal{H}_{n}$-module, and accordingly there are well-behaved induction and restriction functors $\uparrow^{\mathcal{H}_{n+\kappa}}$ and $\downarrow_{\mathcal{H}_{n}}$ between the module categories of these two algebras. If $B$ and $C$ are blocks of $\mathcal{H}_{n}$ and $\mathcal{H}_{n+\kappa}$ as above, then we define $\uparrow^{C}$ to be the composition of $\uparrow^{\mathcal{H}_{n+\kappa}}$ with projection onto $C$, and we define $\downarrow_{B}$ similarly. Now suppose $\lambda$ is an $e$-regular partition in $B$. We define the signature of $\lambda$ to be the sequence of signs obtained by examining runners $j$ and $k$ from bottom to top, writing a + every time there is a bead on runner $k$ with no bead immediately to the right, and a - every time there is a bead on runner $j$ with no bead immediately to the left. We define the reduced signature by successively deleting all adjacent pairs +-, and we say that $\lambda$ is non-exceptional if there are no - signs in the reduced signature. 
In this case, there will be exactly $\kappa+$ signs, and we define the partition $\lambda^{+}$by moving the beads corresponding to these + signs each one place to the right.

\section{Proposition 2.8.}

1. If $\lambda$ is non-exceptional, then $\lambda^{+}$is an e-regular partition lying in $C$, and we have

$$
D^{\lambda} \uparrow^{C} \cong\left(D^{\lambda^{+}}\right)^{\oplus \kappa !}, \quad D^{\lambda^{+}} \downarrow_{B} \cong\left(D^{\lambda}\right)^{\oplus \kappa !} .
$$

2. If $\lambda$ and $\mu$ are two non-exceptional e-regular partitions in $B$, then the $(\lambda, \mu)$-entry of the adjustment matrix for $B$ equals the $\left(\lambda^{+}, \mu^{+}\right)$-entry of the adjustment matrix for $C$.

\section{Proof.}

1. This is an instance of the 'modular branching rules', which are surveyed in [2].

2. This is proved in [10, Lemma 4.3(2)]; although the result is stated there for blocks of weight 3, the proof works generally.

Next we define the pyramid of a block $B$ : take an abacus for $B$, number the runners as above, and for any $0 \leqslant i<j \leqslant e-1$, define the integer

$$
{ }_{i} B_{j}=\left\{\begin{array}{r}
\text { the number of beads on runner } j \text { minus the number of beads on runner } i \\
\text { (if runner } j \text { lies to the right of runner } i \text { ) } \\
\text { the number of beads on runner } j \text { minus the number of beads on runner } i \text { minus } 1 \\
\text { (if runner } j \text { lies to the left of runner } i \text { ). }
\end{array}\right.
$$

The collection $\left({ }_{i} B_{j}\right)$ is called the pyramid for $B$. This was defined by Richards in [24] in a slightly different way: he defined integers ${ }_{i} a_{j}$ which are related to ours by ${ }_{i} a_{j}=\max \left\{w-1-{ }_{i} B_{j}, 0\right\}$, where $w$ is the weight of $B$.

We say that a block $B$ of weight $w$ is Rouquier if we have ${ }_{i} B_{j} \geqslant w-1$ for every $i, j$. The favourable properties of Rouquier blocks have been discussed at length elsewhere (see [4] for a great deal of information about Rouquier blocks of the symmetric groups); we shall need to use them briefly later on. For now, we note that we can get from any block to a Rouquier block via a sequence of Scopes pairs.

Lemma 2.9. [8, Lemma 3.1] Suppose $B$ is a block of $\mathcal{H}_{n}$ of weight $w$. Then there is a sequence of integers $n=n_{0}, \ldots, n_{t}$ and sequence of blocks $B=B_{0}, \ldots, B_{t}$ such that:

- $B_{i}$ is a block of $\mathcal{H}_{n_{i}}$ for $i=0, \ldots, t$;

- $B_{i-1}$ and $B_{i}$ form $a\left[w: n_{i}-n_{i-1}\right]$-pair, for $i=1, \ldots, t$;

- $B_{t}$ is a Rouquier block.

\subsection{Other theorems concerning decomposition numbers}

We shall need to use some other theorems concerning decomposition numbers for Iwahori-Hecke algebras and $q$-Schur algebras.

The Jantzen-Schaper formula (or rather its $q$-analogue, proved by James and Mathas [18, Theorem 4.3]) gives bounds for the decomposition number $\left[S^{\lambda}: D^{\mu}\right]$, provided that the decomposition numbers $\left[S^{v}: D^{\mu}\right]$ are known for partitions $v \triangleright \lambda$. We shall use only the following very special case. 
Proposition 2.10. Suppose $\lambda$ and $\mu$ are partitions of $n$ with the same e-core and with $\mu \triangleright \lambda$, and suppose that there is no other partition $\xi$ of $n$ with this e-core such that $\mu \triangleright \xi \triangleright \lambda$. Given abacus displays for $\lambda$ and $\mu$, suppose that there is exactly one way to get from the abacus for $\lambda$ to the abacus for $\mu$ by moving one bead up its runner and another bead down its runner. Then $\left[W^{\lambda}: L^{\mu}\right]>0$.

Another way to phrase the condition on the abacus displays in this proposition is to say that there are exactly two beta-numbers for $\lambda$ which are not beta-numbers for $\mu$, and these two numbers are incongruent modulo $e$. This interpretation will be helpful later when we use the proposition with $e$ replaced by ep.

A corollary of the Jantzen-Schaper formula is the Carter Criterion [23, Proposition 5.40], which gives the classification of irreducible Specht modules labelled by e-regular partitions. In the case where the underlying field has infinite characteristic, this has a particularly simple statement. We use the following version, in terms of the abacus and the $e$-quotient of a partition; this statement is a special case of [8, Proposition 2.1].

Theorem 2.11. Suppose $\mathbb{F}$ has infinite characteristic, and $\lambda$ is a partition lying in a block $B$ of $\mathcal{H}_{n}$. Suppose that $\lambda[0]=\cdots=\lambda[e-2]=\varnothing$ and $\lambda[e-1]$ has at most ${ }_{e-2} B_{e-1}+1$ non-zero parts. Then $\lambda$ is e-regular and the Specht module $S^{\lambda}$ is irreducible, i.e. $S^{\lambda} \cong D^{\lambda}$.

We also need the decomposition numbers for two-part partitions and two-column partitions, i.e. the decomposition numbers $\left[W^{\lambda}: L^{\mu}\right]$, where $\lambda$ and $\mu$ are partitions both of of the form $(n-a, a)$ or both of the form $\left(2^{a}, 1^{n-2 a}\right)$. For two-part partitions these were computed by James [17, Theorem 20.6], while for two-column partitions they follow from the paper by Thams [25]. Such decomposition numbers are all either 0 or 1 ; we shall need only the case where $e=2$ and the underlying characteristic is infinite.

Theorem 2.12. Suppose $\lambda=(n-a, a)$ and $\mu=(n-b, b)$. Then

$$
d_{\lambda \mu}^{2}=d_{\mu^{\prime} \lambda^{\prime}}^{2}= \begin{cases}1 & (a=b) \\ 1 & (a=b+1 \text { and } n \text { is even }) \\ 0 & \text { (otherwise }) .\end{cases}
$$

Finally, we need the runner removal theorems which relate the decomposition numbers of IwahoriHecke algebras and $q$-Schur algebras over fields of infinite characteristic at different roots of unity. There are two versions.

Theorem 2.13. [19, Theorem 2.2] Suppose $e \geqslant 3$, and $\lambda$ and $\mu$ are partitions of $n$ with the same $e$-core. Take abacus displays for $\lambda$ and $\mu$, and suppose that there is a runner $i$ such that in both abacus displays the last bead on runner $i$ occurs before the first empty space on any runner. Delete runner $i$ from each display, and let $\lambda^{-}$ and $\mu^{-}$be the partitions defined by the resulting abacus displays. Then

$$
d_{\lambda \mu}^{e}=d_{\lambda^{-} \mu^{-}}^{e-1}
$$

Theorem 2.14. [12, Theorem 4.1] Suppose $e \geqslant 3$, and $\lambda$ and $\mu$ are partitions of $n$ with the same e-core. Take abacus displays for $\lambda$ and $\mu$, and suppose that there is a runner $i$ such that in both abacus displays the first empty space on runner $i$ occurs after the last bead on any runner. Delete runner $i$ from each display, and let $\lambda^{-}$ and $\mu^{-}$be the partitions defined by the resulting abacus displays. Then

$$
d_{\lambda \mu}^{e}=d_{\lambda^{-} \mu^{-}}^{e-1}
$$


Example. Suppose $e=4$, and

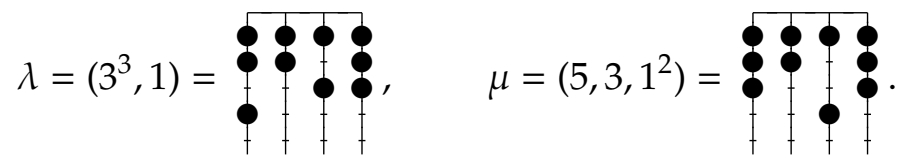

Then we have $d_{\lambda \mu}^{4}=d_{\lambda^{-} \mu^{-}}^{3}$, where we can take either

$$
\lambda^{-}=\left(2^{3}, 1\right)=\begin{aligned}
& 8: \\
& \vdots ; \\
& i f t
\end{aligned}, \quad \mu^{-}=\left(3,2,1^{2}\right)=8 ; 8
$$

using Theorem 2.13, or

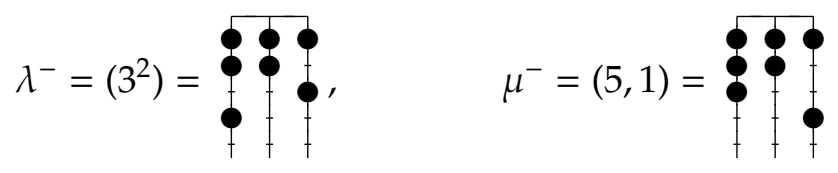

using Theorem 2.14 .

\section{An extension of James's Conjecture}

Now we can state our extension of James's Conjecture. We assume from now on that the characteristic of $\mathbb{F}$ is a prime $p$.

\section{Conjecture 3.1.}

1. Suppose $B$ is a block of the Iwahori-Hecke algebra $\mathcal{H}_{n}$ of weight $w$. Then the adjustment matrix for $B$ is the identity matrix if and only if either $w=p$ and ${ }_{i-1} B_{i}=0$ for all $1 \leqslant i \leqslant e-1$, or $w<p$.

2. Suppose B is a block of the $q$-Schur algebra $\mathcal{S}_{n}$ of weight $w$. Then the adjustment matrix for $B$ is equal to the identity matrix if and only if $w<p$.

In the remainder of the paper, we prove the 'only if' parts of this conjecture. For (1), this is done in Propositions 4.1 and 5.1, and for (2) in Proposition 6.1. The 'if' part of (2) is simply James's Conjecture, but the 'if' part of (1) is a somewhat stronger statement. This statement may be verified for blocks of weight at most 4 using the fact $[24,10,11]$ that James's Conjecture holds for these small weights, together with the explicit adjustment matrices for blocks of weight 2 and 3 in [9] and [13]. We hope the reader will find this evidence persuasive.

\section{Iwahori-Hecke algebras in the case $w>p$}

In this section, we prove the following result.

Proposition 4.1. Suppose $B$ is a block of $\mathcal{H}_{n}$ of weight $w>p$. Then the adjustment matrix for $B$ is not the identity matrix.

Proof. We consider separately the cases where ${ }_{e-2} B_{e-1}=0$ and ${ }_{e-2} B_{e-1}>0$. 
For the case where ${ }_{e-2} B_{e-1}=0$, we define three partitions $\lambda, \mu, v$ in $B$. If $w+p$ is odd, then we set $s=\frac{w-p-1}{2}$ and $t=\frac{w+p+1}{2}$, and define

$$
\lambda=\left\langle e-2_{(s+1)}, e-1_{(t-1)}\right\rangle, \quad \mu=\left\langle e-2_{(s)}, e-1_{(t)}\right\rangle, \quad v=\left\langle e-2_{(t)}, e-1_{(s)}\right\rangle ;
$$

if $w+p$ is even, then we set $s=\frac{w-p}{2}, t=\frac{w+p}{2}$ and define

$$
\lambda=\left\langle e-2_{(t)}, e-1_{(s)}\right\rangle, \quad \mu=\left\langle e-2_{(t+1)}, e-1_{(s-1)}\right\rangle, \quad v=\left\langle e-2_{(s)}, e-1_{(t)}\right\rangle .
$$

In either case, $\lambda$ and $v$ both have the same ep-core, and $v$ is the most dominant partition with this $e p$-core which is dominated by $\mu$. So if we can show that $d_{v \mu}^{e}>0$ and $d_{\lambda \mu}^{e}<d_{\lambda v^{\prime}}^{e p}$ the adjustment matrix for $B$ will be non-trivial, by Lemma 2.7(2).

We can easily check these statements. By Proposition 2.10 we get $d_{\lambda v}^{e p}>0$, and by applying Theorem $2.13 e-2$ times and using Theorem 2.12, we get $d_{\nu \mu}^{e}=1, d_{\lambda \mu}^{e}=0$, which proves the result.

Now we turn to the case where $e_{-2} B_{e-1}>0$. First we need to deal separately with the case where $w=3$ (and $p=2$ ); here we can read from the explicit adjustment matrices for weight three blocks in [13] that the $(\lambda, \mu)$-entry of the adjustment matrix is 1 when

$$
\lambda=\left\{\begin{array}{ll}
\left\langle e-1_{\left(1^{3}\right)}\right\rangle & \left({ }_{e-2} B_{e-1} \geqslant 2\right) \\
\left\langle e-2_{(3)}\right\rangle & \left({ }_{e-2} B_{e-1}=1\right),
\end{array} \quad \mu=\left\langle e-1_{(3)}\right\rangle .\right.
$$

So we assume that $w>3$. We set $p^{\prime}=p$ if $p$ is odd, or $p^{\prime}=4$ if $p=2$. We shall define partitions $\lambda$ and $\mu$ in $B$ with $\mu$ e-regular, such that $d_{\lambda \mu}^{e}=0$ while $d_{\lambda \mu}^{e p^{\prime}}>0$; we can then appeal to Lemma 2.7(1).

If $w \neq \equiv-1\left(\bmod p^{\prime}\right)$, then we set

$$
\lambda=\left\{\begin{array}{ll}
\left\langle e-1_{\left(w-p^{\prime}, p^{\prime}\right)}\right\rangle & \left(w \geqslant 2 p^{\prime}\right) \\
\left\langle e-1_{\left(p^{\prime}-1, w-p^{\prime}+1\right)}\right\rangle & \left(w \leqslant 2 p^{\prime}-2\right),
\end{array} \quad \mu=\left\langle e-1_{(w)}\right\rangle .\right.
$$

If $w \equiv-1\left(\bmod p^{\prime}\right)$, then we set

$$
\lambda=\left\{\begin{array}{ll}
\left\langle e-1_{\left(w-p^{\prime}-1, p^{\prime}+1\right)}\right\rangle & \left(w \geqslant 3 p^{\prime}-1\right) \\
\left\langle e-1_{\left(p^{\prime}, p^{\prime}-1\right)}\right\rangle & \left(w=2 p^{\prime}-1\right),
\end{array} \quad \mu=\left\langle e-1_{(w-1,1)}\right\rangle .\right.
$$

It is easy to see that $\lambda$ and $\mu$ have the same $e p^{\prime}$-core, and that there is no $\xi$ with this $e p^{\prime}$-core for which $\mu \triangleright \xi \triangleright \lambda$. Hence we can use Proposition 2.10 to get $d_{\lambda \mu}^{e p^{\prime}}>0$; note that the conditions of that proposition really are satisfied - the two beta-numbers of $\lambda$ which are not beta-numbers of $\mu$ are incongruent modulo $e p^{\prime}$ (though they are congruent modulo $e$ ). On the other hand, Theorem 2.11 implies that $d_{\lambda \xi}^{e}=\delta_{\lambda \xi}$ for any $\xi$; in particular, $d_{\lambda \mu}^{e}=0$, and so by Lemma 2.7(1) the theorem is proved.

Examples. We provide examples to help the reader unravel the notation in the above proof.

1. First suppose that $B$ is the 3 -block of the symmetric group $\Xi_{28}$ with core $\left(2,1^{2}\right)$. Thus we have $e=p=3$ and $w=8$. $B$ has an abacus in which the numbers of beads on the runners are $8,10,9$ from left to right, and therefore the pyramid for $B$ satisfies ${ }_{0} B_{1}=1,{ }_{1} B_{2}=0,{ }_{0} B_{2}=2$. We define

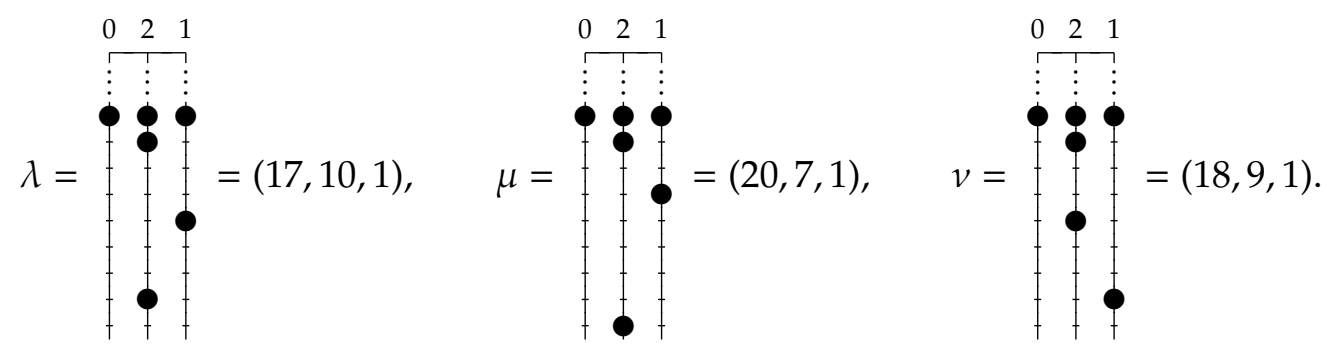


$\lambda$ and $v$ both have 9-core $\left(8,1^{2}\right)$, while $\mu$ has 9-core $(6,3,1)$. It is easy to check that $d_{\lambda v}^{9}>1$, using Proposition 2.10. To calculate $d_{\lambda \mu}^{3}$ and $d_{v \mu}^{3}$, we remove runner 0 from each abacus display to leave the partitions

$$
\lambda^{-}=(10,6), \quad \mu^{-}=(12,4), \quad v^{-}=(11,5) .
$$

We have $d_{\lambda \mu}^{3}=d_{\lambda^{-} \mu^{-}}^{2}=0$ and $d_{v \mu}^{3}=d_{v^{-} \mu^{-}}^{2}=1$, from Theorems 2.12 and 2.13.

2. Now suppose $e=5$ and $p=2$, and that $B$ is the block of $\mathcal{H}_{44}$ with core $\left(5,1^{4}\right)$. $B$ has an abacus in which the numbers of beads on the runners are 7, 8, 8, 8,9 from left to right, and we find that ${ }_{3} B_{4}=1$. We set
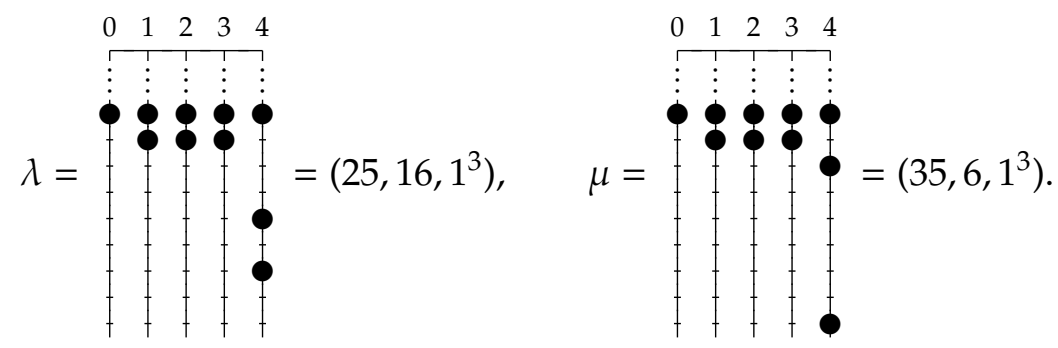

Then $d_{\lambda \mu}^{5}=0$, because by Theorem $2.11 \lambda$ labels an irreducible Specht module in infinite characteristic, while $d_{\lambda \mu}^{20}>0$ by the Jantzen-Schaper formula.

\section{Iwahori-Hecke algebras in the case $w=p$}

The case $w=p$ is delicate, since some blocks of weight $p$ have non-trivial adjustment matrices, but some do not. Our main result is the following.

Proposition 5.1. Suppose $B$ is a block of $\mathcal{H}_{n}$ of weight $p$, and that for some $1 \leqslant i \leqslant e-1$ we have ${ }_{i-1} B_{i}>0$. Then the adjustment matrix of $B$ is not the identity matrix.

In order to prove this, we explicitly construct two partitions which we claim give a non-zero off-diagonal entry in the adjustment matrix. We then prove this claim by inducing the corresponding simple modules up to a Rouquier block.

In order to define our partitions, we introduce an operation on abacus displays. Take an abacus display for a partition $\mu$, and for each $j \in\{0, \ldots, e-1\}$ let $p_{j}$ be the position of the lowest bead on runner $j$. Now given $i \in\{0, \ldots, e-1\}$, let $\hat{p}=\min \left\{p_{i}, p_{i+1}, \ldots, p_{e-1}\right\}$. Define the partition $s_{i}(\mu)$ by moving the bead at position $\hat{p}$ down one space.

Now suppose $B$ is a weight $p$ block of $\mathcal{H}_{n}$, and that ${ }_{i-1} B_{i}>0$ for some $i$; take an abacus for $B$, and suppose that the numbers of beads on the runners are $b_{0}, \ldots, b_{e-1}$ from left to right. Let

$$
\tilde{\mu}=\left\langle\mid b_{0}, \ldots, b_{e-1}\right\rangle,
$$

i.e. the core of $B$, and let

$$
\tilde{\lambda}=\left\langle i_{\left(1^{2}\right)} \mid b_{0}, \ldots, b_{e-1}\right\rangle,
$$

a partition of weight 2 with the same core as $B$. Now set

$$
\begin{aligned}
& \mu_{B}^{i}=s_{i}^{p}(\tilde{\mu}), \\
& \lambda_{B}^{i}=s_{i}^{p-2}(\tilde{\lambda}) .
\end{aligned}
$$

Since the function $s_{i}$ obviously preserves the core of a partition and increases its weight by 1 , both $\lambda_{B}^{i}$ and $\mu_{B}^{i}$ lie in $B$. 
Example. Suppose $e=6$ and $p=7$, and let $B$ be the block with core $\left(19,14,9^{2}, 5^{2}, 4,2^{4}\right)$. This has an abacus display with $\left(b_{0}, \ldots, b_{5}\right)=(8,10,9,11,13,7)$ :

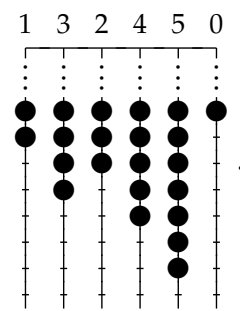

Taking $i=2$, we get the following abacus displays for $\mu_{B}^{i}$ and $\lambda_{B}^{i}$ :
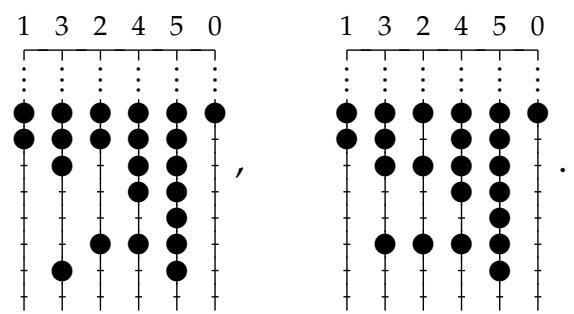

So we have

$$
\begin{aligned}
& \mu_{B}^{i}=\left\langle 2_{(3)}, 3_{(3)}, 4_{(1)} \mid 8,10,9,11,13,7\right\rangle=\left(19,17,15^{3}, 12,7^{2}, 3^{2}, 2\right), \\
& \lambda_{B}^{i}=\left\langle 2_{(3,1)}, 3_{(2)}, 4_{(1)} \mid 8,10,9,11,13,7\right\rangle=\left(19,14^{4}, 12,7^{2}, 3^{4}, 1^{2}\right) .
\end{aligned}
$$

Proposition 5.1 will follow immediately from the next result.

Proposition 5.2. Suppose $B$ is as above. Then $\lambda_{B}^{i} \neq \mu_{B^{\prime}}^{i}$ and the $\left(\lambda_{B^{\prime}}^{i} \mu_{B}^{i}\right)$-entry of the adjustment matrix for $B$ is 1 .

In order to prove this, we use downwards induction via Scopes pairs, as follows.

Lemma 5.3. Suppose $B$ and $C$ are weight $p$ blocks forming $a[p: \kappa]$-pair, and that there exists $1 \leqslant i \leqslant e-1$ such that ${ }_{i-1} B_{i}>0$. Then the partitions $\lambda_{B^{\prime}}^{i} \mu_{B^{\prime}}^{i} \lambda_{C}^{i}$ and $\mu_{C}^{i}$ are all e-regular, and $\lambda_{B}^{i}$ and $\mu_{B}^{i}$ are non-exceptional for the pair $(B, C)$, with

$$
\begin{aligned}
& D^{\lambda_{B}^{i} \uparrow C} \cong\left(D^{\lambda_{C}^{i}}\right)^{\oplus \kappa !}, \quad D^{\lambda_{C}^{i} \downarrow_{B}} \cong\left(D^{\lambda_{B}^{i}}\right)^{\oplus \kappa !}, \\
& D^{\mu_{B}^{i} \uparrow}{ }^{C} \cong\left(D^{\mu_{C}^{i}}\right)^{\oplus \kappa !}, \quad D^{\mu_{C}^{i}} \downarrow_{B} \cong\left(D^{\mu_{B}^{i}}\right)^{\oplus \kappa !} \text {. }
\end{aligned}
$$

Hence the $\left(\lambda_{B^{\prime}}^{i}, \mu_{B}^{i}\right)$-entry of the adjustment matrix for B equals the $\left(\lambda_{C^{\prime}}^{i} \mu_{C}^{i}\right)$-entry of the adjustment matrix for C.

Example. Continuing the last example, let $C$ be the weight 7 block with core $\left(19,14,9^{2}, 5^{3}, 2^{4}\right)$. This has an abacus with $\left(b_{0}, \ldots, b_{5}\right)=(8,9,10,11,13,7)$, and $B$ and $C$ form a [7:1]-pair. We have
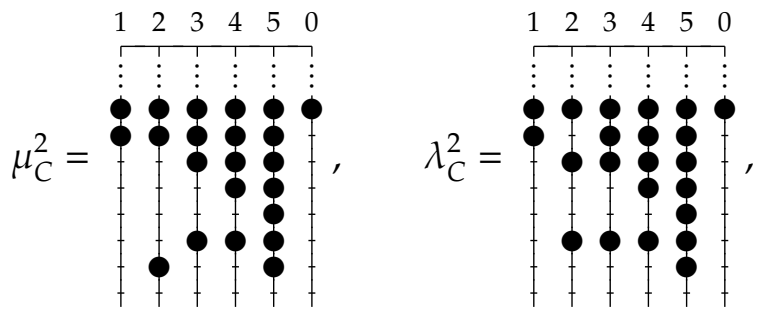

and we may verify Lemma 5.3 using Proposition 2.8 and the discussion preceding it. 
Proof of Lemma 5.3. Suppose that an abacus for $C$ is obtained from an abacus for $B$ by swapping runners $j$ and $k$, where $j<k$ (so runner $k$ lies immediately to the left of runner $j$ in the abacus for $B$ ).

First we consider the partition $\xi=\mu_{B}^{i}$. The abacus display for this partition has the following properties:

1. if $l<i$, then $\xi[l]=\varnothing$;

2. if $l \geqslant i$, then $\xi[l]$ has at most one non-zero part;

3. if $l<m$ and $\xi[m]=\varnothing$, then the first empty space on runner $m$ occurs after the lowest bead on runner $l$;

4. if both $\xi[l]$ and $\xi[m]$ are non-empty, then the positions of the lowest beads on runners $l$ and $m$ differ by less than $e$;

5. $\xi$ has weight $p$.

Moreover, these properties determine $\xi$ uniquely. This may easily be proved inductively: one can show that for any $j$, properties $1-4$ uniquely specify a partition of weight $j$ (namely, $s_{i}^{j}(\tilde{\mu})$ ).

Given properties 1 and 2, we can show that $\xi$ is $e$-regular. For if it is not, then there is sequence of $e$ consecutive positions on the abacus all occupied by beads, with at least one empty space occurring before these beads. One of these positions must lie on runner 0 , but properties 1 and 2 together with the fact that $i>0$ imply that the last bead on runner 0 occurs before the first empty space on any runner; contradiction.

Now we use properties 1-4 to find all the possible configurations of runners $j$ and $k$ in the abacus for $\xi$, and work out the signature. In each case, the signature of $\xi$ consists either of just $\kappa+$ signs, or of +- followed by $\kappa+$ signs. Either way, $\xi$ is non-exceptional, so there is a partition $\xi^{+}$in $C$ such that

$$
D^{\xi} \uparrow^{C} \cong\left(D^{\xi^{+}}\right)^{\oplus \kappa !}, \quad D^{\xi^{+}} \downarrow_{B} \cong\left(D^{\xi}\right)^{\oplus \kappa !} .
$$

Moreover, it will be apparent from a comparison of the abacus displays for $\xi$ and $\xi^{+}$that properties 1-5 hold for $\xi^{+}$too, so (by the above statement that properties that these properties uniquely determine a partition) we must have $\xi^{+}=\mu_{C}^{i}$.

There are five possibilities for the configuration of runners $j$ and $k$, as follows:
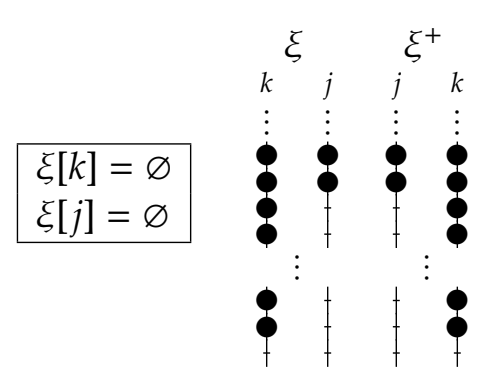
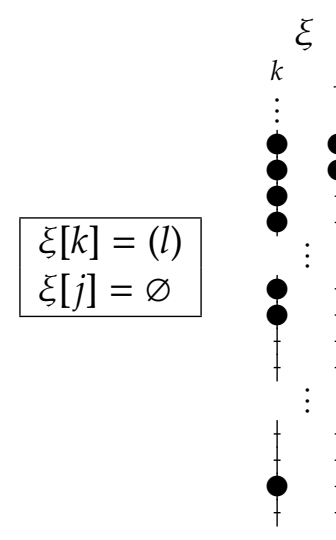

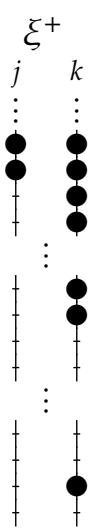

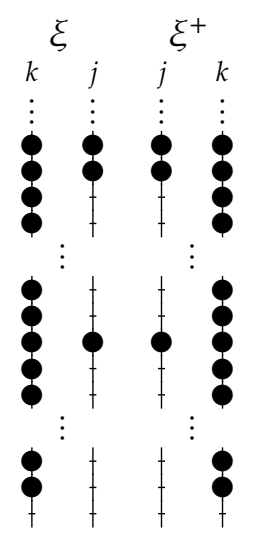




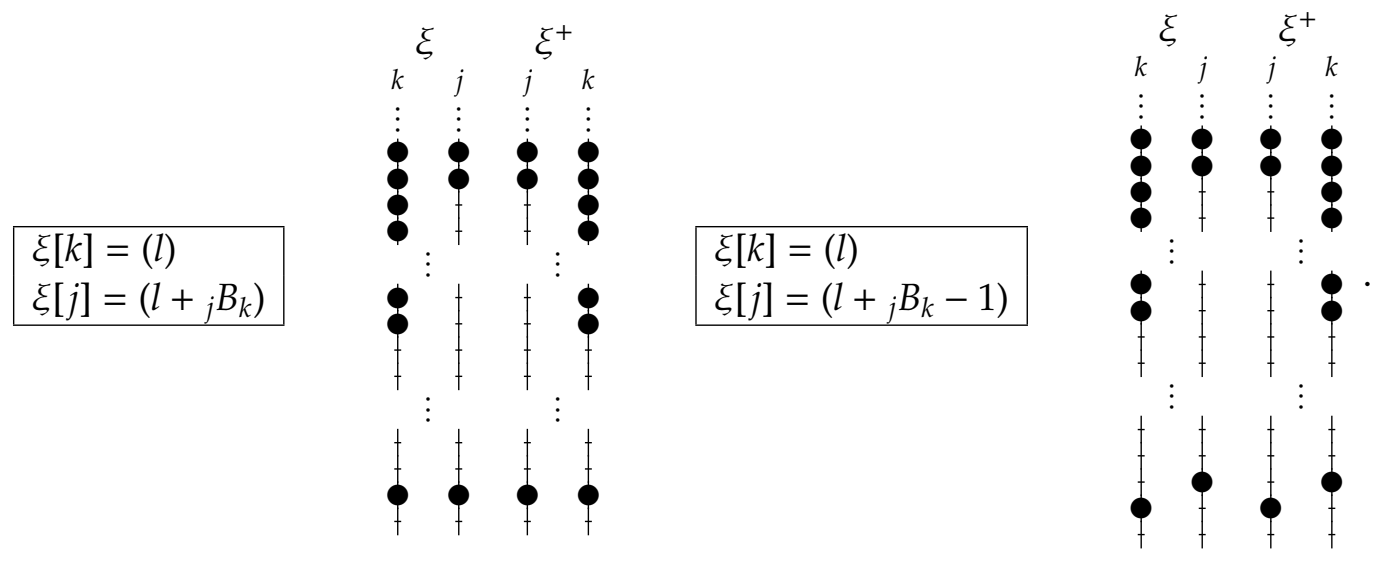

The result follows for $\mu_{B}^{i}$. For the partition $\xi=\lambda_{B}^{i}$, we follow the same line of proof, with slightly different details; here property 2 must be replaced with

2. if $l>i$, then $\xi[l]$ has at most one non-zero part, while if $l=i$ then $\xi[l]$ has the form $(r, 1)$ for some $r$.

The $e$-regularity of $\lambda_{B}^{i}$ follows in the same way as the $e$-regularity of $\mu_{B}^{i}$; the fact that ${ }_{0} B_{i}>0$ ensures the condition that the last bead on runner 0 occurs before the first empty space on any runner. For the induction to $C$, there are four additional possibilities for the abacus display, which arise if $j$ or $k$ equals $i$ :

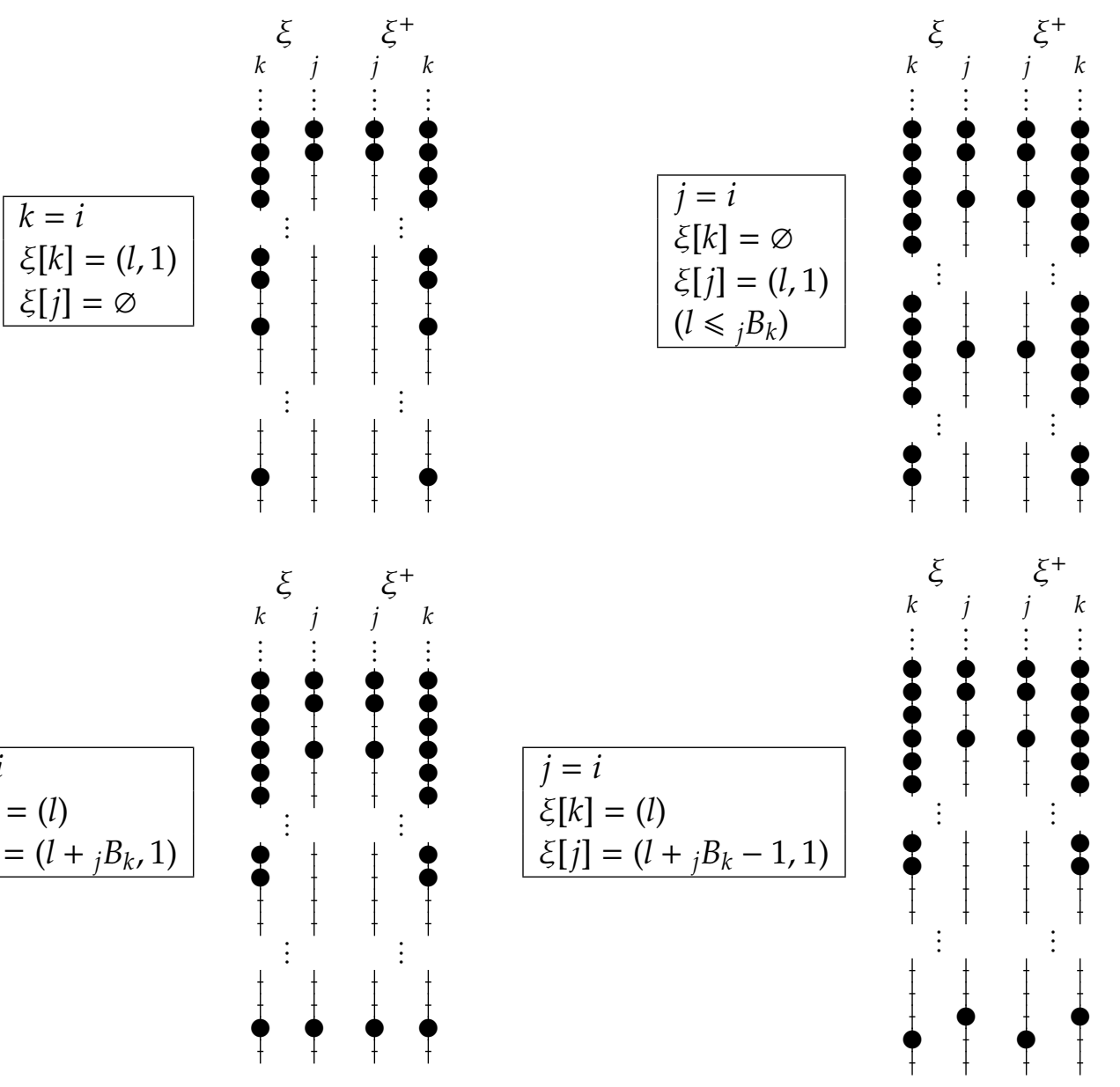


Note that the condition ${ }_{i-1} B_{i}>0$ guarantees that the configuration

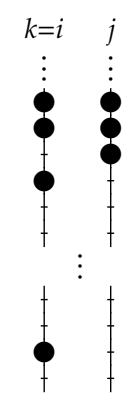

cannot occur in $\xi$; so we really can use the same reasoning as for the partition $\mu_{B^{\prime}}^{i}$, and the result is proved.

Using Lemma 5.3 and Lemma 2.9, we need only prove Proposition 5.2 for Rouquier blocks; in this case, the result is straightforward.

Lemma 5.4. Suppose B is a Rouquier block of $\mathcal{H}_{n}$ of weight $p$. Then we have

$$
\lambda_{B}^{i}=\left\langle i_{(p-1,1)}\right\rangle, \quad \mu_{B}^{i}=\left\langle i_{(p)}\right\rangle,
$$

and Proposition 5.2 holds for B.

Proof. Since ${ }_{i} B_{i+1} \geqslant p-1$, the function $s_{i}$ will always move the lowest bead on runner $i$ down one space; so the partitions $\mu_{B}^{i}$ and $\lambda_{B}^{i}$ are as claimed.

To show that the $\left(\lambda_{B^{\prime}}^{i} \mu_{B}^{i}\right)$-entry of the adjustment matrix is non-zero, we examine $d_{\lambda \mu}^{e}$ and $d_{\lambda \mu^{\prime}}^{e p}$ where $\lambda=\lambda_{B}^{i}$ and $\mu=\mu_{B}^{i}$. By applying Theorem 2.14e-1-i times and then using Theorem 2.11 (or by using the known decomposition numbers for Rouquier blocks in infinite characteristic [3, 21]), we find that $d_{\lambda \mu}^{e}=0$; by Proposition 2.10 (or by using the well-known decomposition numbers for blocks of weight 1), we get $d_{\lambda \mu}^{e p}>0$. Lemma 2.7(1) now implies that there is some $\xi$ in $B$ with $\mu \triangleright \xi \unrhd \lambda$ such that the $(\xi, \mu)$-entry of the adjustment matrix for $B$ is non-zero. But it is a simple matter to check that there is no partition in $B$ lying strictly between $\mu$ and $\lambda$ in the dominance order, so $\xi=\lambda$ and the result is proved.

We can now immediately deduce Proposition 5.2 and hence Proposition 5.1.

\section{6 q-Schur algebras}

In this section, we deal with blocks of the $q$-Schur algebra. Given our work on Iwahori-Hecke algebras, we are already most of the way to proving our main result.

Proposition 6.1. Suppose B is a block of the $q$-Schur algebra $\mathcal{S}_{n}$ of weight $w \geqslant p$. Then the adjustment matrix for $B$ is not the identity matrix.

Proof. Let $B^{\prime}$ be the block of $\mathcal{H}_{n}$ with the same core as $B$. Then the adjustment matrix for $B^{\prime}$ occurs as a submatrix of the adjustment matrix for $B$, so the result is true when $w>p$ or when ${ }_{0} B_{1}>0$, by Propositions 4.1 and 5.1. So we assume that $w=p$ and ${ }_{0} B_{1}=0$. We define

$$
\lambda=\left\langle 0_{\left(1^{p}\right)}\right\rangle, \quad \mu=\left\langle 0_{\left(1^{p-1}\right)}, 1_{(1)}\right\rangle, \quad v=\left\langle 1_{\left(1^{p}\right)}\right\rangle .
$$


Now $v$ lies in an ep-block of weight 1 , and furthermore is the most dominant partition in this block which is dominated by $\mu$. So by Lemma 2.7(2) it suffices to show that $d_{\nu \mu}^{e}>0$ and $d_{\lambda v}^{e p}>d_{\lambda \mu}^{e}$. Proposition 2.10 yields $d_{\lambda v}^{e p}>0$, while for the decomposition numbers $d_{\nu \mu}^{e}$ and $d_{\lambda \mu^{\prime}}^{e}$ we may use Theorem $2.14 e-2$ times to reduce to the case $e=2$; in this case the partitions are
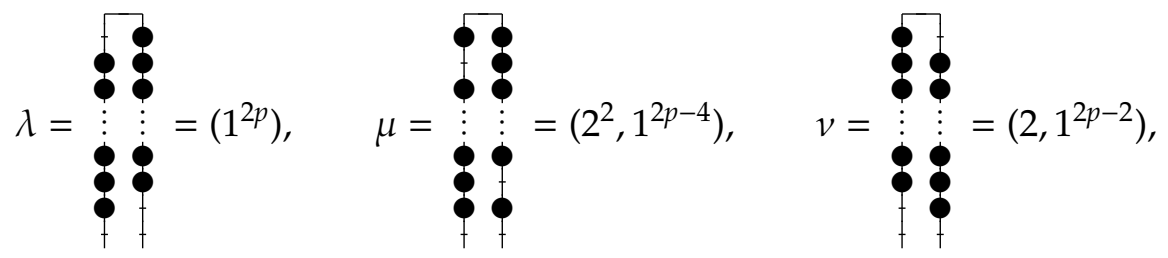

and by Theorem 2.12 we get

$$
d_{v \mu}^{e}=1, \quad d_{\lambda \mu}^{e}=0
$$

Remark. Proposition 6.1 does not give a complete picture for $q$-Schur algebras. We have chosen to restrict attention to the Schur algebra $\mathcal{S}_{\mathbb{F}, q}(n, n)$, but one could ask which blocks of the $q$-Schur algebra $\mathcal{S}_{\mathbb{F}, q}(N, n)$ have trivial adjustment matrix, when $N$ and $n$ are arbitrary. The answer for $N>n$ will be the same as for $N=n$, but for $N<n$ the situation is more complicated: here there only exist Weyl modules and simple modules corresponding to partitions $\lambda$ with $\lambda_{1} \leqslant N$, and the classification of the blocks (due to Cox [5]) is not so straightforward. We hope to address this question in future.

\section{More counterexamples to Rouquier's conjecture}

In personal communication, Raphaël Rouquier described to the author his conjectured algorithm for computing the decomposition matrices of the symmetric groups, and the counterexample to this conjecture which arose after some computation; this conjecture is also mentioned in [15, Example 3.2]. The conjecture suggests that the decomposition numbers should be the smallest possible integers which are consistent with all decomposition maps from Iwahori-Hecke algebras in infinite characteristic, i.e. with Theorem 2.6 for all values of $i$. Rouquier's own counterexample to this conjecture arose for the principal block of the symmetric group $\mathfrak{S}_{13}$ in characteristic 2 . The left-hand matrix below gives the adjustment matrix predicted by Rouquier's conjecture for this block, while the matrix on the right is the correct adjustment matrix.

$$
\begin{array}{l|lllllllllll}
(13) & 1 & . & . & . & . & . & . & . & . & . & . \\
(11,2) & 1 & 1 & . & . & . & . & . & . & . & . & . \\
(10,2,1) & . & . & 1 & . & . & . & . & . & . & . & . \\
(9,4) & 1 & 1 & . & 1 & . & . & . & . & . & . & . \\
(9,3,1) & 2 & . & . & . & 1 & . & . & . & . & . & . \\
(8,4,1) & . & . & . & . & . & . & . & . & . & . & . \\
(7,6) & 1 & . & . & 1 & . & . & . & . & . & . & . \\
(7,5,1) & . & . & . & . & . & . & 1 & . & . & . & . \\
(7,4,2) & 1 & 1 & . & 1 & . & . & . & 1 & . & . & . \\
(6,4,3) & 2 & . & 1 & . & . & . & . & . & 1 & . & . \\
(5,4, & . & . & . & . \\
& 4 & . & . & . & . & . & . & & & .
\end{array}
$$

$(5,4,3,1) \quad 4$

$\mid \begin{array}{lllllllllll}1 & . & . & . & . & . & . & . & . & . & . \\ 1 & 1 & . & . & . & . & . & . & . & . & . \\ . & . & 1 & . & . & . & . & . & . & . & . \\ 1 & 1 & . & 1 & . & . & . & . & . & . & . \\ 2 & . & . & . & 1 & . & . & . & . & . & . \\ 1 & . & . & . & . & . & . & . & . & . & . \\ 1 & . & . & 1 & . & . & . & . & . & . & . \\ . & . & . & . & . & . & 1 & . & . & . & . \\ 1 & 1 & . & 1 & . & . & . & 1 & . & . & . \\ 2 & . & 1 & . & . & . & 1 & . & 1 & . & . \\ 4 & 2 & . & . & . & . & . & . & . & 1 & . \\ 4 & . & . & . & 1\end{array}$


Although Rouquier's conjecture is false, it is natural to ask in the context of the present paper whether the following special case is true: that the adjustment matrix for a block is the identity matrix if and only if this is consistent with Theorem 2.6. Unfortunately, this too is false; there are blocks for which Rouquier's conjecture suggests a trivial adjustment matrix, but for which the results of this paper show that the adjustment matrix is non-trivial. So we have further, and in some sense simpler, counterexamples to Rouquier's conjecture. The first such example (extending the conjecture to blocks of Iwahori-Hecke algebras generally, rather than just symmetric group algebras) occurs with $e=4$ and $p=3$, with $B$ being the weight 3 block of $\mathcal{H}_{16}$ with 4 -core $\left(2^{2}\right)$. It can be checked that an adjustment matrix equal to the identity would be consistent with Theorem 2.6. (What this says is that for any $i$ there is a matrix $A^{(i)}$ with non-negative integer entries such that $D_{B}^{(4)}=D_{B}^{\left(4.3^{i}\right)} A^{(i)}$. In fact, this needs checking only for $i=1$, since for $i \geqslant 2$ the matrix $D^{\left(4.3^{i}\right)}$ is the identity matrix.) However, the pyramid of $B$ satisfies ${ }_{1} B_{2}=1$, so by Proposition $5.1 B$ has non-trivial adjustment matrix; in fact, the $\left(\left(6^{2}, 3,1\right),(9,7)\right)$-entry is non-zero.

Of course, this example could have been observed from [13]. But the results of this paper provide a wealth of such counterexamples; in particular, there are more counterexamples in the symmetric group case. The first such occurs in characteristic 5 , for the block of $\mathfrak{S}_{31}$ with 5 -core $\left(3^{2}\right)$.

In both Rouquier's counterexample and ours, the adjustment matrix entries which are not explained by Theorem 2.6 can be explained by semi-simple induction and restriction of simple modules. In Rouquier's case, the troublesome $((8,4,1),(13))$-entry of the adjustment matrix must equal the $((8,4),(12))$-entry of the adjustment matrix for $\Im_{12}$ in characteristic 2 by Proposition 2.8 , and one can show using Theorem 2.6 that this entry is non-zero. In our case, we found non-trivial entries of adjustment matrices by restricting simple modules from Rouquier blocks. So a way to rescue Rouquier's conjecture might be to examine all blocks of a given weight simultaneously, and to hope that the entries of the adjustment matrix for any block might be the smallest integers consistent with Theorem 2.6 and with Proposition 2.8. Not having checked this suggestion against all known adjustment matrices, the author refrains from calling it a conjecture.

\section{References}

[1] S. Ariki, 'On the decomposition numbers of the Hecke algebra of $G(m, 1, n)^{\prime}$, J. Math. Kyoto Univ. 36 (1996), 789-808.

[2] J. Brundan \& A. Kleshchev, 'Representation theory of the symmetric groups and their double covers', Groups, combinatorics \& geometry (Durham, 2001), 31-53, World Sci. Publishing, River Edge, NJ, 2003.

[3] J. Chuang \& K. M. Tan, 'Some canonical basis vectors in the basic $U_{q}\left(\widehat{\mathfrak{s l}}_{n}\right)$-module', J. Algebra 248 (2002), 765-779.

[4] J. Chuang \& K. M. Tan, 'Filtrations in Rouquier blocks of symmetric groups and Schur algebras', Proc. London Math. Soc (3) 86 (2003), 685-706.

[5] A. Cox, 'The blocks of the q-Schur algebra', J. Algebra 207 (1998), 306-325.

[6] R. Dipper \& G. James, 'Representations of Hecke algebras of general linear groups', Proc. London Math. Soc. (3) 52 (1986), 20-52.

[7] R. Dipper \& G. James, 'q-tensor space and q-Weyl modules', Trans. Amer. Math. Soc. 327 (1991), 251-82. 
[8] M. Fayers, 'Irreducible Specht modules for Hecke algebras of type A', Adv. Math. 193 (2005), $438-452$.

[9] M. Fayers, 'Weight two blocks of Iwahori-Hecke algebras in characteristic two', Math. Proc. Cambridge Philos. Soc. 139 (2005), 385-397.

[10] M. Fayers, 'Decomposition numbers for weight three blocks of symmetric groups and IwahoriHecke algebras', Trans. Amer. Math. Soc. 360 (2008), 1341-1376.

[11] M. Fayers, 'James's Conjecture holds for weight four blocks of Iwahori-Hecke algebras', J. Algebra 317 (2007), 593-633.

[12] M. Fayers, 'Another runner removal theorem for $v$-decomposition numbers of Iwahori-Hecke algebras and q-Schur algebras', J. Algebra 310 (2007), 396-404.

[13] M. Fayers \& K. M. Tan, 'Adjustment matrices for weight three blocks of Iwahori-Hecke algebras', J. Algebra 306 (2006), 76-103.

[14] M. Geck, ‘Brauer trees of Hecke algebras', Comm. Algebra 20 (1992), 2937-2973.

[15] M. Geck, 'Representations of Hecke algebras at roots of unity', Astérisque 252 (1998), 33-55.

[16] J. Graham \& G. Lehrer, ‘Cellular algebras', Invent. Math. 123 (1996), 1-34.

[17] G. James, Representation of general linear groups, LMS Lecture Notes 94, Cambridge University Press, 1984.

[18] G. James \& A. Mathas, 'A q-analogue of the Jantzen-Schaper theorem', Proc. London Math. Soc. (3) 74 (1997), 241-274.

[19] G. James \& A. Mathas, 'Equating decomposition numbers for different primes', J. Algebra 258 (2002), 599-614.

[20] A. Lascoux, B. Leclerc \& J.-Y. Thibon, 'Hecke algebras at roots of unity and crystal bases of quantum affine algebras', Comm. Math. Phys. 181 (1996), 205-263.

[21] B. Leclerc \& H. Miyachi, 'Some closed formulas for canonical bases of Fock spaces', Represent. Theory 6 (2002), 290-312.

[22] B. Leclerc \& J.-Y. Thibon, 'Littlewood-Richardson coefficients and Kazhdan-Lusztig polynomials', Advanced Studies in Pure Mathematics 28 (2000), 155-220.

[23] A. Mathas, Iwahori-Hecke algebras and Schur algebras of the symmetric group, University lecture series 15, American Mathematical Society, Providence, RI, 1999.

[24] M. Richards, 'Some decomposition numbers for Hecke algebras of general linear groups', Math. Proc. Cambridge Philos. Soc. 119 (1996), 383-402.

[25] L. Thams, 'The submodule structure of the quantum symmetric powers', Bull. Austral. Math. Soc. 50 (1994), 29-39.

[26] M. Varagnolo \& E. Vasserot, 'On the decomposition matrices of the quantized Schur algebra', Duke J. Math. 100 (1999), 267-297. 